\title{
A NOTE ON SEMI-GROUPS IN A LOCALLY COMPACT GROUP
}

\author{
ANATOLE BECK
}

1. Introduction. In a recent paper by this author and others [1], the following theorem is proved:

Theorem 3 (Simon). In a compact group, every semi-group which contains a set of positive measure is an open and closed subgroup and therefore is itself measurable.

In this paper, we show that this result can be improved to the following:

THEOREM A. In a locally compact group, every semi-group of nonzero inner measure and finite outer measure is an open compact subgroup.

This theorem can also be used to show an elusive ${ }^{1}$ point in Theorem 5 of $[1]$.

2. Proof of Theorem A. We rely heavily in this proof on Theorem 1 of [1], which states:

Theorem 1. Let $G$ be a locally compact topological group with completed Haar measure $\mu$ and outer measure $\mu^{*}$. Let $A, B \subset G$ be sets such that $\mu(A)>0$ and $\mu^{*}(B)>0$. Then the interior of $B A$ (also $\left.A B\right)$ is nonempty.

Let $S$ now be a semi-group in a locally compact group $G$ with Haar measure $\mu$. We assume that $\mu_{*}(S)>0$, so that $S$ contains a measurable set of nonzero measure. Thus, by Theorem 1, the interior of $S^{2} \subset S$ is nonempty. Hence $S_{0}$, the interior of $S$, is also nonempty. Since $S$ has finite outer measure, $S_{0}$ is measurable of finite measure. It is also clear that $S_{0} \cdot S \subset S_{0}$. For each $s \in S_{0}$, we have

$$
s \cdot S_{0} \subset S_{0}^{2} \subset S_{0} \cdot S \subset S_{0},
$$

so that, since $\mu$ is left invariant,

$$
\mu\left(S_{0}\right)=\mu\left(s \cdot S_{0}\right) \leqq \mu\left(S_{0}^{2}\right) \leqq \mu\left(S_{0}\right) .
$$

Received by the editors February 10, 1960.

1 The author expresses his debt to Mr. John E. Lange for pointing out this elusiveness. 


\section{Therefore}

$$
\mu\left(S_{0} \backslash s \cdot S_{0}\right)=0 .
$$

Since $s \cdot S_{0} \subset s \cdot \bar{S}_{0}$, we see that $\mu\left(S_{0} \backslash s \cdot \bar{S}_{0}\right)=0$. But $S_{0} \backslash s \bar{S}_{0}$ is open, so that it must be empty, and $s \cdot \bar{S}_{0} \supset S_{0}$. We now set $U=s^{-1} \cdot S_{0}$. This is clearly a neighborhood of the identity in $G$, and we have $s \cdot \bar{S}_{0} \supset S_{0} \supset S_{0}$ $\cdot S_{0}=s \cdot U \cdot S_{0} \supset s \cdot \bar{S}_{0}$. Thus, $S_{0}=s \cdot \bar{S}_{0}$, and $S_{0}$ is closed, so that $S_{0}$ $=s \cdot S_{0}$.

It follows immediately that $S_{0}$ is an open and closed subgroup of $G$. We now have

$$
S=e \cdot S \subset S_{0} \cdot S \subset S_{0} \subset S .
$$

Therefore $S=S_{0}$. Since $S$ is an open and closed subgroup of finite measure, $S$ is compact.

3. Proof of part of Theorem 5 in [1]. Part of Theorem 5 of [1] requires the proof that if in a locally compact group $G$ we have two measurable sets $T$ and $S$ with $T$ a subgroup and $S$ a sub-semigroup of $G$, and if $\mu(T)=\infty$ and $\mu(T \backslash S)<\infty$, then we have $T \subset S$. We construct the set $A=T \cap\left(S \backslash S^{-1}\right)$; then we observe that $A^{-1}$ is a semigroup, for if $a_{1}, a_{2} \in A$, then $a_{2} a_{1} \in S$. However, $a_{1}^{-1} a_{2}^{-1} \in S$, for that would give us $a_{2}^{-1} \in a_{1} S \subset S$, and $a_{2} \in S^{-1}$, contrary to assertion. Thus, $a_{1}^{-1} a_{2}^{-1} \in A^{-1}$, which is thus a semi-group. Since $A^{-1}=T \cap\left(S^{-1} \backslash S\right)$, we see that $\mu\left(A^{-1}\right) \leqq \mu(T \backslash S)<\infty$. If $\mu\left(A^{-1}\right)>0$, we have, by Theorem A, that $A^{-1}$ is an open subgroup, and thus contains $e$, which is false, since $A \cap A^{-1}=\square$. Thus $\mu(A)=0$, and $T \cap S \cap S^{-1}$, which is a subgroup of $T$, has the additional property that

$$
\begin{aligned}
\mu\left(T \cap S \cap S^{-1}\right) & =\infty, \\
\mu\left(T \backslash\left(S \cap S^{-1}\right)\right) & =\mu(T \backslash S)+\mu(A)<\infty .
\end{aligned}
$$

It now follows easily (cf. [1, Lemma 5.1]) that $T=\left(S \cap S^{-1}\right) \cap T$, which gives us $T \subset S$.

\section{BIBLIOGRAPHY}

1. Anatole Beck, H. H. Corson and A. B. Simon, The interior points of the product of two subsets of a locally compact group, Proc. Amer. Math. Soc. vol. 9 (1958) pp. 648652.

UNIVERSITY OF WISCONSIN 